\title{
Second-harmonic generation with zero group-velocity mismatch in nonlinear photonic crystal fibers
}

Bache, Morten; Lægsgaard, Jesper; Bang, Ole; Bjarklev, Anders Overgaard

Published in:

Proceedings ICTON 2006

Link to article, DOI:

10.1109/ICTON.2006.248320

Publication date:

2006

Document Version

Publisher's PDF, also known as Version of record

Link back to DTU Orbit

Citation (APA):

Bache, M., Lægsgaard, J., Bang, O., \& Bjarklev, A. O. (2006). Second-harmonic generation with zero groupvelocity mismatch in nonlinear photonic crystal fibers. In Proceedings ICTON 2006 (Vol. 2, pp. 49-54). IEEE. https://doi.org/10.1109/ICTON.2006.248320

\section{General rights}

Copyright and moral rights for the publications made accessible in the public portal are retained by the authors and/or other copyright owners and it is a condition of accessing publications that users recognise and abide by the legal requirements associated with these rights.

- Users may download and print one copy of any publication from the public portal for the purpose of private study or research.

- You may not further distribute the material or use it for any profit-making activity or commercial gain

- You may freely distribute the URL identifying the publication in the public portal 


\title{
Second-Harmonic Generation with Zero Group-Velocity Mismatch in Nonlinear Photonic Crystal Fibers
}

\author{
Morten Bache, Jesper Lagsgaard, Ole Bang and Anders Bjarklev \\ COM•DTU Department of Communications, Optics \& Materials, Technical University of Denmark, Bld. 345v, \\ DK-2800 Lyngby, Denmark \\ Tel: +454525 3775,Fax:+4545936581,e-mail: bache@com.dtu.dk
}

\begin{abstract}
We consider an index-guiding silica photonic crystal fiber with a triangular hole-pattern and a periodically poled quadratic nonlinearity. By tuning the pitch and the relative size of the holes, second-harmonic generation with zero group-velocity mismatch is found to be feasible for any fundamental wavelength above $780 \mathrm{~nm}$. The phasevelocity mismatch has a lower limit with coherence lengths in the micron range. The nonlinear strength is optimized when the fundamental has maximum confinement in the core. The conversion bandwidth allows for fs-pulse conversion and $4-180 \% /\left(\mathrm{W} \cdot \mathrm{cm}^{2}\right)$ relative efficiencies were found.
\end{abstract}

Keywords: second-harmonic generation, photonic crystal fibers, dispersion control, conversion of ultra-short pulses.

\section{INTRODUCTION}

Relying on quadratic nonlinearities, second-harmonic generation (SHG) is widely used for efficient wavelength conversion devices in order to extend the spectral range of laser sources and to do all-optical wavelength multiplexing. Efficient conversion from the fundamental to the second-harmonic ( $\mathrm{SH}$ ) mode requires a small phase mismatch between them. Phase matching to the lowest order is typically achieved through a quasi-phase matching (QPM) technique [1], whereby the group-velocity mismatch (GVM) sets the limits to device length and bandwidth for pulsed SHG. Zero GVM is therefore crucial when converting ultra-short pulses in the ps range or below. For SHG in fibers, zero GVM for restricted wavelengths was predicted by changing the core radius of a conventional step-index fiber [2] and by using mode-matching [3]. For bulk media zero GVM was found for restricted wavelengths by spectrally noncritical phase matching [4] and by combining non-collinear QPM with a pulse-front tilt [5].

Here we investigate efficient pulsed SHG in a silica photonic crystal fiber (PCF), having a standard indexguiding triangular design with a single rod defect in the center. The PCF design is sketched in the inset of Fig. 1. The main design parameters of the PCF are the pitch $\Lambda$ and the relative hole-diameter $D=d / \Lambda$. The nonlinearity is thought to be induced, e.g., by thermal poling as has recently been demonstrated in PCFs [6].

As is well known (and as shown later), the SHG conversion efficiency, defined as the output SH power $P_{2, \text { out }}$ relative to the input fundamental power $P_{1, i n}$, is

$$
\eta=P_{2, \text { out }} / P_{1, \text { in }} \propto P_{1, \text { in }} \rho^{2} l_{F}^{2} \operatorname{sinc}^{2}\left(\Delta \beta l_{F} / 2\right) .
$$

Efficient SHG can therefore be achieved by increasing $P_{1, i n}$, as well as by having a large nonlinear material response and a strong modal overlap, which both are included in the $\rho$ parameter. The phase mismatch between the fundamental and SH modes is $\Delta \beta=2 \beta_{1}-\beta_{2}$, where $\beta_{j}$ is the mode propagation constant. To the lowest order, the coherence length $l_{c o h}=\pi / \Delta \beta$ gives the range (typically microns) over which power is exchanged efficiently to the $\mathrm{SH}$, after which the power flow reverses. This is a serious limitation to the conversion efficiency, but a QPM grating can compensate for this. If we assume that this is the case, then we encounter the problem of also achieving phase matching for higher orders. As mentioned above, this is especially important when converting ultra-short transform limited Gaussian pulses, because for such a pulse with duration $\tau$, the SHG bandwidth $\Delta \lambda$ required for conversion is $\Delta \lambda=2 \ln (2) \lambda_{1}{ }^{2} /(\pi c \tau)$. Neglecting the weak frequency dependence on $\rho, \Delta \lambda$ is determined by the width of $\operatorname{sinc}^{2}\left(\Delta \beta l_{F} / 2\right)$. Thus, taking a small fiber length $l_{F}$ gives a large $\Delta \lambda$ but also a poor efficiency because $\eta$ scales as $l_{F}{ }^{2}$. We will instead minimize $\Delta \beta$, whose first order contribution is GVM: thus, zero GVM gives larger bandwidths. Also, GVM implies a temporal walk-off length $l_{W}$ between the modes so we are forced to take $l_{F}<l_{W}$. When GVM is zero, $l_{W}$ diverges and $l_{F}$ can be taken longer, thereby increasing $\eta$ dramatically. Zero GVM is thus important both as to be able to convert short pulses as well as to increase the conversion efficiency.

PCFs are interesting candidates for efficient fiber SHG due to their flexibility concerning dispersion design [7] and maximizing the nonlinear strength [8]. Previous investigations [9] of SHG in PCFs considered the scalar case and found large bandwidths and strong modal overlaps for selected parameter values. Instead, we perform a detailed vectorial analysis over a continuous parameter space, and show zero GVM for any fundamental

The work reported in this paper was supported in part by The Danish Natural Science Research Council (FNU, grant no. 21-04-0506). 
wavelength $\lambda_{1}>780 \mathrm{~nm}$ by merely adjusting $\Lambda$ and $D$. This is a much simpler way of removing GVM compared to previous methods [3-5], it promises very large bandwidths due to its flexibility, and it is very efficient. The other interesting aspect of PCFs is their ability to guide light in a very small core resulting in very large effective nonlinearities. We will show that the nonlinear SHG strength scales as the ratio $D / \Lambda$, and that it reaches its maximum when the fundamental is maximally confined in a core. This happens when the pitch $\Lambda$ reaches a value roughly comparable to the fundamental wavelength.

\section{DISPERSION CALCULATIONS}

A fiber mode can be described by an effective index $n=c / v_{p h}$, i.e., the ratio of the speed of light $c$ to the phase velocity of the mode $v_{p h}=\omega / \beta$. The dispersive character of the propagation constant $\beta$ gives a phase-velocity mismatch between the fundamental $\left(\omega_{1}\right)$ and $\mathrm{SH}\left(\omega_{2}=2 \omega_{1}\right)$ modes, which we classify through the index mismatch $\Delta n=n_{1}-n_{2}=c\left[1 / v_{p h}\left(\omega_{1}\right)-1 / v_{p h}\left(\omega_{2}\right)\right]=c\left(\beta_{1} / \omega_{1}-\beta_{2} / \omega_{2}\right)$, related to the phase mismatch $\Delta \beta$ as $\Delta n=\Delta \beta \lambda_{1} / 4 \pi$. The index mismatch is obviously a measure of the difference in phase velocities between the modes. The group velocity is instead defined as $1 / v_{g}=\partial \beta / \partial \omega$, giving a GVM (walk-off) parameter $d_{12}=1 / v_{g}\left(\omega_{1}\right)-1 / v_{g}\left(\omega_{2}\right)$, describing the difference in group velocities between the modes.

We calculated the transverse fiber modes with the MIT Photonic-Bands (MPB) package [10]. Each unit cell contained $n_{C}{ }^{2}=32^{2}$ grid points, and the super cell contained $n_{S C}{ }^{2}=5^{2}$ unit cells. In the MPB programme we first calculated the fundamental mode frequency and group velocity, followed by iterations of the SH until $\left|\omega_{2}-2 \omega_{1}\right|<10^{-8}$. Material dispersion, parameterized by the silica Sellmeier equation [11], was then included using a perturbative technique [12], whose advantage is that many different values of the pitch $\Lambda$ can be calculated perturbatively from the MPB data (where $\Lambda$ is unity.)

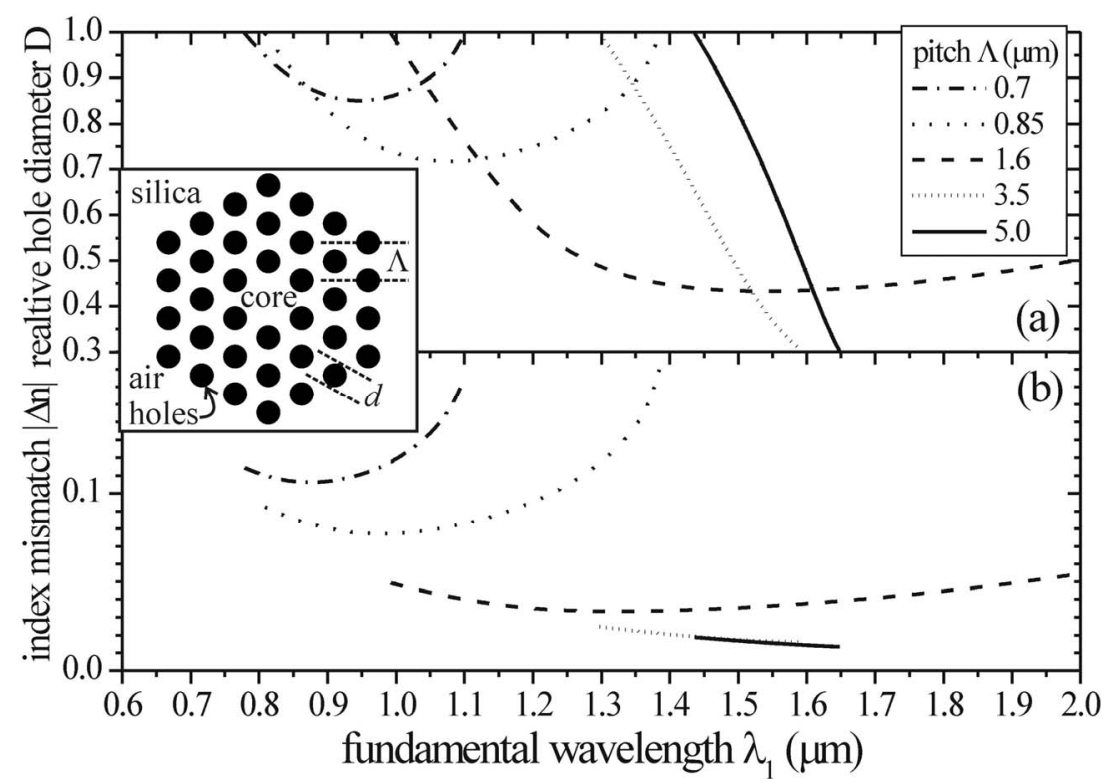

Figure 1. (a) Zero GVM contours in $\left(\lambda_{1}, D\right)$-space for $\Lambda$ fixed, and (b) $|\Delta n|$ along these contours. Inset: Sketch of the considered triangular index-guiding silica PCF with a hole pitch $\Lambda$ and air-hole diameter $d$. A single-hole defect defines the core.

The extracted group velocities from the MPB data and the perturbation calculations can now be plotted in a suitable parameter space. What we try to look for is designs where the GVM parameter $d_{12}=0$. This we have done in a suitable chosen parameter space consisting of the limits $0.6 \leq \lambda_{1} \leq 2.0 \mu \mathrm{m}$ and $0.3 \leq D \leq 1$. (The first limit is chosen because below $\lambda_{1}=0.6$ the Sellmeier equation breaks down for the SH wavelength. The second limit was chosen because PCFs with $D<0.3$ are hard to make, while $D>1$ is unphysical in the present design.) We kept the pitch $\Lambda$ fixed and then tried to locate the contour in this $2 \mathrm{D}$ space where $d_{12}=0$. The result is shown in Fig. 1a. The examples show that the design parameters can be tuned over a continuous range to achieve zero GVM for any $\lambda_{1}>0.78 \mu \mathrm{m}$. In Fig. $1 \mathrm{~b}$ we show that the corresponding index mismatch $\Delta n$ is never zero. This is a general trend, even with non-zero GVM, so a QPM method is needed to achieve lowest order phase-matching. In Fig. $1 \mathrm{~b}$ we also note that for $\Lambda=0.70,0.85$ and $1.6 \mu \mathrm{m}$ a minimum in $\Delta n$ appears around $\lambda_{1} \approx \Lambda$. We checked that the core diameter $d_{c} \approx \Lambda(2-D)$ relative to $\lambda_{1}$ is maximum there. Thus, when $\lambda_{1}$ increases beyond this point, $d_{c} / \lambda_{1}$ shrinks so the fundamental is less confined in the core. Since the SH stays well-confined (because $\lambda_{2}=\lambda_{1} / 2$ ), an increase in $|\Delta n|$ follows.

Focusing on the telecom, Nd:YAG, and Ti:Sapphire operating wavelengths $\left(\lambda_{1}=1.55,1.06\right.$, and $0.8 \mu \mathrm{m}$, respectively) Fig. 2a shows the $D$-value required to get zero GVM as $\Lambda$ is changed. For $\lambda_{1}=0.80 \mu \mathrm{m}$ zero GVM 
requires very large $D$-values, e.g., $D=0.96$ for $\Lambda=0.70 \mu \mathrm{m}$. For such $D$-values deviations from the ideal circular holes must be expected, which might influence the results. Instead, for both $\lambda_{1}=1.06$ and $1.55 \mu \mathrm{m}$ the lowest required $D$-values are in a range where the ideal round holes should be preserved. In Fig. $2 \mathrm{~b}$ we have calculated the SHG bandwidth $\Delta \lambda$ by expanding $\Delta \beta$ around $\lambda_{1}$ up to third order

$$
\Delta \beta\left(\lambda_{1}+\Delta \lambda\right) \approx \sum_{m=0}^{3} \frac{\Delta \lambda^{m}}{m !} \frac{\partial^{m}}{\partial \lambda^{m}} \Delta \beta\left(\lambda_{1}\right)
$$

The bandwidth $\Delta \lambda$ is then found as the FWHM of the $\operatorname{sinc}^{2}$-term in equation (1). The dominating contribution is the $m=0$ term, which we assume that a QPM grating can compensate for (as in conventional fibers [2]). As discussed before, in this case GVM is the next dominating term, but since we have $d_{12}=0$, the 2nd order dispersion dominates yielding very large bandwidths. Moreover, because $d_{12}=0, \Delta \lambda$ of a fiber with length $l_{F}$ scales as $l_{F}^{-1 / 2}$ (instead of scaling as $l_{F}^{-1}$ when $\left|d_{12}\right|>0$ ), so a longer device can be created without loosing too much bandwidth. The large peaks in $\Delta \lambda$ around $\Lambda=1 \mu \mathrm{m}$ for $\lambda_{1}=1.06 \mu \mathrm{m}$ and $\Lambda=1.6 \mu \mathrm{m}$ for $\lambda_{1}=1.55 \mu \mathrm{m}$ can be explained from Fig. 1a: Exactly for these values of the pitch a minimum in the zero GVM contour occurs, implying that the 2 nd order term vanishes and very large $\Delta \lambda$ results (large enough for fs pulse conversion). In other words, at this point also the mismatch between the group-velocity dispersion (GVD) of each mode is vanishing. It is important to stress that the modes do not have zero GVD here, but only that the GVD mismatch between the fundamental and the SH is zero. Thus, the zero GVD mismatch observed here is different from what dispersion control in PCFs are usually used for, namely to obtain a dispersion flattened fiber, such as in, e.g., Ref. [7]. Finally, Fig.2c shows the QPM period $l_{Q P M}=2\left|l_{c o h}\right|=\lambda_{1} /(2|\Delta n|)$ required to achieve lowest order phase matching [1]. Such values can be reached using periodic poling: either by writing the periodic pattern using photolithography on the electrode placed on the flat part of a D-shaped fiber [2], or by using laser ablation on an electrode deposited in the fiber [13].

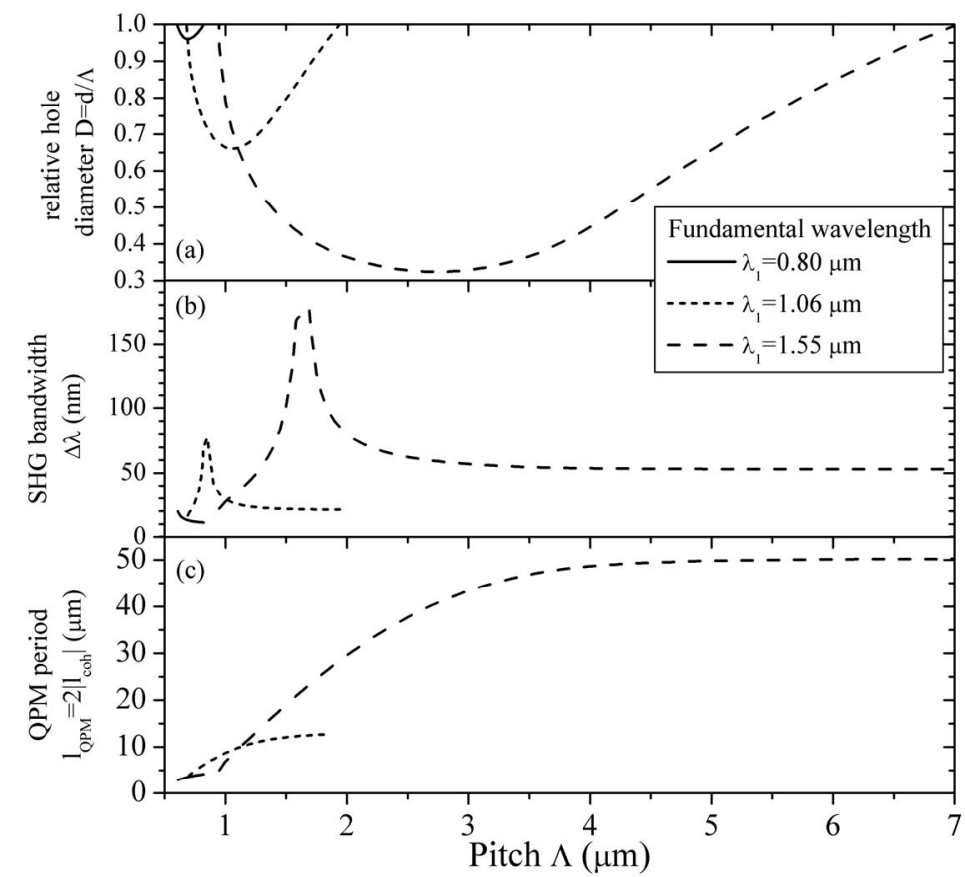

Figure 2. (a) Zero GVM contours in $(\Lambda, D)$-space and fixing $\lambda_{I}$. (b) and (c) $\Delta \lambda$ and $l_{Q P M}$ along these contours.

\section{EFFECTIVE NONLINEARITY}

Using the reductive perturbation method [14], the nonlinear dimensionless equations for SHG in the frame of reference traveling with the group velocity of the fundamental are

$$
\begin{aligned}
& \left(\frac{\partial}{\partial z}-i D_{1} \frac{\partial^{2}}{\partial t^{2}}\right) u_{1}=i \sigma u_{1}^{*} u_{2} e^{-i \Delta \beta z l_{F}}, \\
& \left(\frac{\partial}{\partial z}-d_{12} \frac{l_{F}}{\tau} \frac{\partial}{\partial t}-i D_{2} \frac{\partial^{2}}{\partial t^{2}}\right) u_{2}=i \frac{\sigma}{2} u_{1}^{2} e^{i \Delta \beta z l_{F}},
\end{aligned}
$$

We have here assumed that the nonlinearity is weak so we may take $\mathbf{E}_{j}(z, \mathbf{x}, t)=A_{j}(z, t) \mathbf{B}_{j}(\mathbf{x}) e^{i\left(\beta_{j} z-\omega_{j} t\right)}+$ c.c. where $\mathbf{x}=(x, y)$. We also assume weak transverse variations in the refractive index so $\nabla \times \nabla \times \mathbf{E} \approx-\nabla^{2} \mathbf{E}$, greatly 
simplifying Maxwell's wave equation, which is the starting point of the analysis. The dimensionless propagating fields are found as $u_{j}(z, t)=A_{j}(z, t)\left(N_{j} n_{j} a_{j} c \tau / \Lambda^{2} l_{F}\right)^{1 / 2}$, which conveniently gives $\int d t\left|u_{j}(z, t)\right|^{2}=N_{j}(z)$, where $N_{j}$ is the photon number of the mode. The dimensionless transverse MPB modes are given as $\mathbf{e}_{j}(\mathbf{x})=\mathbf{B}_{j}(\mathbf{x})\left(\varepsilon_{0} \Lambda^{2} l_{F} / 2 \hbar \omega_{j} N_{j}\right)^{1 / 2}$. The coordinate scaling is chosen so $z$ is scaled to $l_{F}$, and $t$ is scaled to the fundamental input pulse length $\tau$. We have also introduced the dimensionless dispersion parameters and the dimensional mode areas (with unit $\mathrm{m}^{2}$ )

$$
\begin{aligned}
& D_{j}=l_{F} /\left(2 \tau^{2}\right) \partial_{\omega}^{2} \beta_{j} \\
& a_{j}=\int d \mathbf{x}\left|\mathbf{e}_{j}(\mathbf{x})\right|^{2}
\end{aligned}
$$

The dimensionless nonlinear parameter is

$$
\sigma=\rho l_{F} \sqrt{2 \hbar \omega_{1}^{2} \omega_{2} / n_{1}^{2} n_{2} \varepsilon_{0} c^{3} \tau}
$$

whose behaviour in the parameter space we will investigate in what follows. It has a very important contribution from the spatial overlap between the material nonlinear tensor $\tilde{\chi}^{(2)}$ and the dimensionless transverse field distributions, which we summarize in the following parameter

$$
\rho=\frac{\left|\int d \mathbf{x} \mathbf{e}_{1}^{*}(\mathbf{x}) \cdot \tilde{\chi}^{(2)}(\mathbf{x}): \mathbf{e}_{2}(\mathbf{x}) \mathbf{e}_{1}^{*}(\mathbf{x})\right|}{\sqrt{a_{1}^{2} a_{2}}}
$$

The modes being vectorial, their overlap cannot be characterized by a single overlap area, but $\rho^{2}$ is equivalent to the scalar expression of Ref. [2] $d_{e f f}^{2} / A_{o v l}$ (the effective nonlinearity squared relative to the effective overlap area.), so the unit of $\rho^{2}$ is $\mathrm{pm}^{2} /\left(\mathrm{V}^{2} \cdot \mathrm{m}^{2}\right)$. The $\tilde{\chi}^{(2)}$ tensor is assumed to have the non-zero elements $\tilde{\chi}_{j j i}^{(2)}=\tilde{\chi}_{j i j}^{(2)}=\tilde{\chi}_{i j j}^{(2)}=\tilde{\chi}_{i i i}^{(2)} / 3$, where $i$ is the main direction of the poling voltage, and $j$ is either of the 2 remaining directions [15]. The considered PCF has two degenerate solutions that are $x$ - and $y$-polarized, respectively, so it will suffice to consider $I=x$. We chose $\tilde{\chi}_{x x x}^{(2)}=1 \mathrm{pm} / \mathrm{V}$, which is feasible by thermal poling of fibers [2].

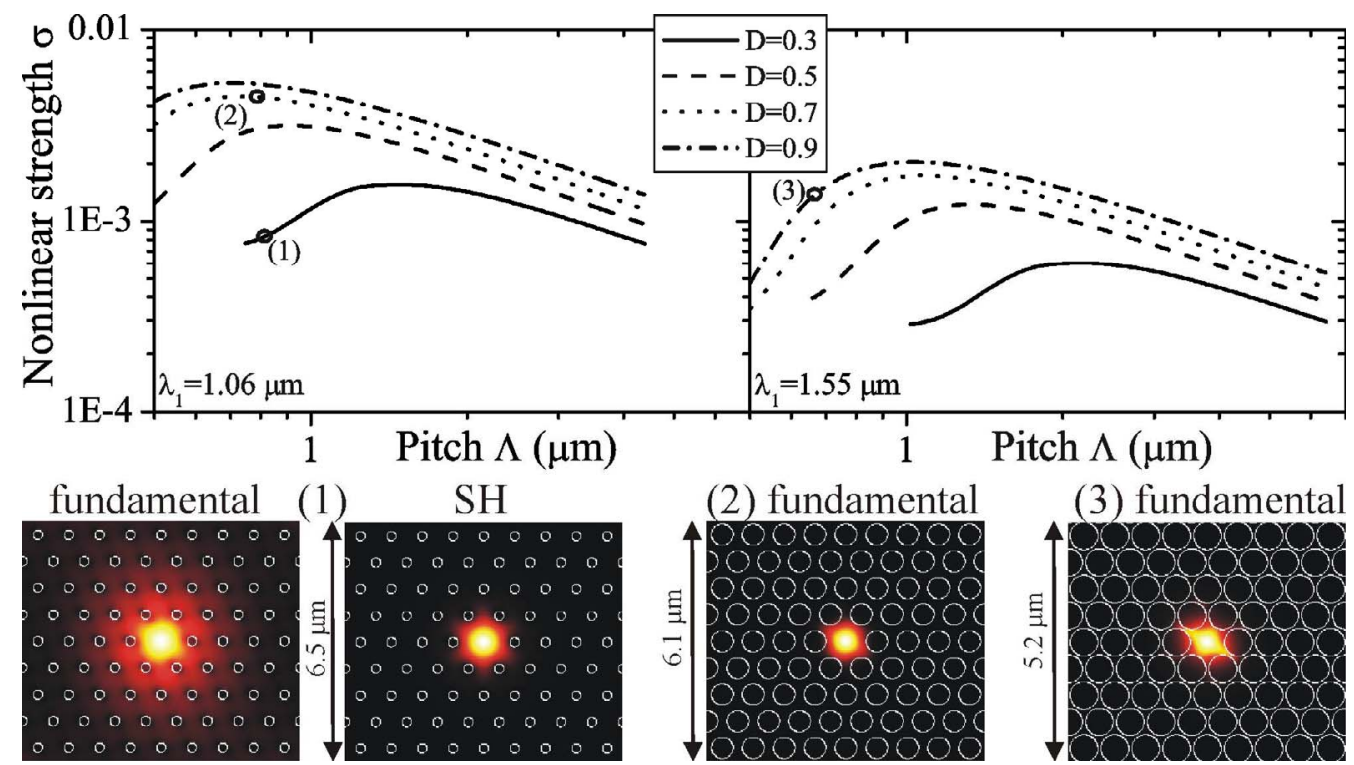

Figure 3. Double-log plots of the nonlinear strength $\sigma$ vs. $\Lambda$ for various values of $D$ and $\lambda_{1}$. (1)-(3): selected mode energy distributions, corresponding to the parameter locations indicated in the plots. $l_{F}=10 \mathrm{~cm}, \tau=1 \mathrm{ps}$.

As usual for nonlinear wave-mixing, the idea is to maximize the overlap between the transverse modes. This implies that effective overlap area must be as small as possible: in other words the optimal situation is that where all of the interacting waves are well confined in the core (not leaking too much into the cladding) and when the mode areas are as small as possible. The core should therefore be as small as possible, until the core reaches a diameter on the order of the wavelength of the guided light, where guidance breaks down: the light starts to leak into the cladding. When mixing waves of different frequencies this is a disaster because the overlap between the different modes becomes very poor. Figure 3 shows the nonlinear strength $\sigma$ for $\lambda_{1}$ and $D$ fixed (note that these curves are not zero-GVM contours.) A $2 / \pi$ reduction of $\chi^{(2)}$ is included because we assume lowest order phase matching through a QPM grating [1]. We found that $\sigma$ scales as $D / \Lambda$, which is due to 
a smaller core when $\Lambda$ is reduced and a better core confinement when $D$ is increased. $\sigma$ peaks when $\Lambda$ takes values around $\lambda_{1}$ (similar to what was observed in [8]), and drops for $\Lambda<\lambda_{1}$ because the fundamental mode has maximum core confinement at the peak [Fig. 3(2)]. It becomes more poorly confined when $\Lambda<\lambda_{1}$, while the SH stays better confined [cf. Fig. 3(1)], resulting in a poor modal overlap (controlled by the $\rho$ parameter). A similar effect gives the minimum in $|\Delta n|$ in Fig. 1b. For large $D$, a decent fundamental mode confinement is observed even for $\Lambda<\lambda_{1}$ [compare Fig. 3(3) with (1),] giving a shift in the peak towards smaller $\Lambda . \sigma$ also increases when $\lambda_{1}$ is reduced because if $d$ and $\Lambda$ are fixed, the light is better confined for smaller $\lambda$.

Table 1 shows data for selected designs of SHG with zero GVM. The 1.06 and $1.55 \mu \mathrm{m}$ designs are chosen to give as large bandwidths as possible, while the $800 \mathrm{~nm}$ design was simply chosen as the lowest $D$-value on the zero GVM contour for any pitch. In order to understand how short a transform-limited pulse these bandwidths are capable of transforming, we report the pulse duration limit $\tau_{\text {lim }}$, as found from the expression $\Delta \lambda=2 \ln (2) \lambda_{1}^{2} /\left(\pi c \tau_{\text {lim }}\right)$. These are well into the fs range, making ultra-short pulse conversion feasible. We checked that the fundamental is single mode for all three designs by using the criterion $\lambda_{1} / \Lambda>2.80(D-0.406)^{0.89}[16]$. For the $1.55 \mu \mathrm{m}$-design also the $\mathrm{SH}$ is single mode. In table 1 we also report the SHG efficiency $\eta=P_{2, \text { out }} / P_{1, \text { in }}$, which can be estimated in the linear regime by solving equation (3) (assuming an undepleted fundamental and neglecting temporal dispersion). Doing this gives

$$
\eta=P_{2, \text { out }} / P_{1, \text { in }}=P_{1, \text { in }} \sigma^{2} \operatorname{sinc}^{2}\left(\Delta \beta l_{F} / 2\right) \frac{\tau}{2 \hbar \omega_{1}}=P_{1, \text { in }} \rho^{2} l_{F}^{2} \operatorname{sinc}^{2}\left(\Delta \beta l_{F} / 2\right) \frac{2 \omega_{1}^{2}}{n_{1}^{2} n_{2} \varepsilon_{0} c^{3}}
$$

$\Delta \beta$ is here evaluated from the fundamental pulse-length and the calculated dispersion. The efficiency is usually reported relative to the input fundamental power, i.e. $\eta / P_{1, \text { in }}$ having the unit $\% / \mathrm{W}$, or even as relative to the fundamental power and the fiber length. The latter we define as the relative efficiency $\eta_{R}=\eta /\left(l_{F}^{2} P_{1, \text { in }}\right)$ having the units $\% /(\mathrm{W} \mathrm{cm})^{2}$. We should here mention that one should be a bit cautious to report the relative efficiency when converting ultra-short pulses where the SHG bandwidth is used to the limit. The reason is that in this case the fiber length affects quite strongly the $\operatorname{sinc}^{2}$-expression and $\eta_{R}$ is therefore no longer independent of $l_{F}$. In the opposite case, the $\operatorname{sinc}^{2}$-expression is close to unity and changes only weakly on the fiber length so $\eta_{R}$ is independent of $l_{F}$, which was the reason in the first place to introduce it. As observed in table 1, very large relative efficiencies are found. For the 1.06 and $1.55 \mu \mathrm{m}$-designs it is mainly because the designs are chosen so the 2 nd order dispersion is zero. This gives very high bandwidths and also a sinc ${ }^{2}$-expression close to unity. For the $800 \mathrm{~nm}$ design we were not able to get zero GVD mismatch, but instead the design has a very large $D$-value which results in a strong confinement, and thereby the spatial mode overlap, in a small core: notice that the core diameter $d_{c}$ for this design is much smaller than for the other designs. We checked that also the two other designs could get larger relative efficiencies by increasing $D$, but instead we have to sacrifice the bandwidth. This should remain as an important conclusion: there is usually a trade-off between bandwidth and effective nonlinearity.

Table 1. Selected designs for SHG with zero GVM. $l_{F}=10 \mathrm{~cm}, \tau=1 \mathrm{ps}, \tilde{\chi}_{x x x}^{(2)}=1 \mathrm{pm} / \mathrm{V}$.

\begin{tabular}{|c|c|c|c|c|c|c|c|c|}
\hline$\lambda_{1}(\mu \mathrm{m})$ & $D$ & $\Lambda(\mu \mathrm{m})$ & $d_{c}(\mu \mathrm{m})$ & $\Delta \lambda(\mathrm{nm})$ & $\tau_{\text {lim }}(\mathrm{fs})$ & $l_{Q P M}(\mu \mathrm{m})$ & $\sigma \cdot 10^{-3}$ & $\eta_{R}\left(\% /\left[\mathrm{W} \cdot \mathrm{cm}^{2}\right]\right)$ \\
\hline 0.80 & 0.96 & 0.70 & 0.73 & 13 & 73 & 4.3 & 10.3 & 183.6 \\
\hline 1.06 & 0.72 & 0.85 & 1.09 & 77 & 21 & 7.7 & 4.4 & 42.3 \\
\hline 1.55 & 0.43 & 1.60 & 2.51 & 170 & 21 & 29.1 & 1.0 & 3.71 \\
\hline
\end{tabular}

\section{MICROSTRUCTURED POLYMER OPTICAL FIBERS AS EFFICIENT SHG DEVICES}

In this paper we considered standard silica PCFs, where the usually centro-symmetric silica material is thought to get a $\chi^{(2)}$ nonlinear response through thermal poling. However, a new class of PCFs have recently appeared made of polymer material, the socalled microstructured polymer optical fibers (mPOFs) [17]. (The reason why they are not called, e.g., polymer PCFs is that standard polymer fibers have traditionally been called POFs.) In our group, we have recently purchased a drawing tower for drawing mPOFs, and have already demonstrated the first mPOF biosensor [18]. The advantages of mPOFs to silica PCFs are many. First of all, mPOFs have a much lower drawing temperature, around $3-400{ }^{\circ} \mathrm{C}$, compared to the $1800-2000{ }^{\circ} \mathrm{C}$ of silica fibers. This implies that the drawing tower equipment is much less expensive. In addition, silica PCFs are produced by drawing a preform made by stacking hollow silica rods. Instead mPOFs are drawn from a solid polymer preform wherein holes have been drilled. Thus, while silica PCFs always have some degree of hexagonal symmetry to its design due to the stacking limitation, mPOFs can have any hole-design, only limited by the drilling technology available. Finally, polymers can have huge nonlinear coefficients, Kerr as well as $\chi^{(2)}$ nonlinearities: the latter can be $2-3$ orders of magnitude larger than in traditional nonlinear glass-types. If one considers equation (7) it emerges that this could give $4-6$ orders of magnitude larger efficiency. The downside of polymers is that they 
have a quite large absorption, especially in the near-IR and UV, where they are virtually non-transparent. The non-vanishing absorption in the visible is not a big problem when short devices are considered, such as the frequency converting fiber we have in mind here. In addition, the absorption around the telecom wavelengths is usually not so big either, so wave-mixing processes with very different wavelengths, such as SHG with $\lambda_{1}=1550 \mathrm{~nm}$ and $\lambda_{2}=775 \mathrm{~nm}$, should be possible despite that the wavelengths in between are heavily absorbed. Of course, it would set a limitation to ultra-short pulse conversion of telecom pulses, where a bandwidth of several $100 \mathrm{~nm}$ is required. Nonetheless, we believe that $\mathrm{mPOFs}$ are interesting candidates for nonlinear fibers for frequency conversion. Preliminary calculations using the Sellmeier equations for the polymer PMMA, which is the most widely chosen material for POFs and mPOFs, show that tuning behaviour similar to what has been demonstrated for silica in this paper is possible.

\section{CONCLUSIONS}

To conclude, by tuning the pitch and relative hole size of an index-guiding silica PCF, SHG with zero GVM could be achieved for any $\lambda_{1}>780 \mathrm{~nm}$. The method holds great promise due to its tunability and simplicity, and conversion bandwidths suitable for fs pulse-conversion were found. The SHG nonlinear strength was optimized when the fundamental was maximally confined, and it was inversely proportional to the pitch and proportional to the relative size of the air holes. We found up to $180 \% /\left(\mathrm{W} \cdot \mathrm{cm}^{2}\right)$ relative conversion efficiencies with a realistic raw $\chi^{(2)}$ nonlinearity of $1 \mathrm{pm} / \mathrm{V}$ and when optimizing for the bandwidth. Even larger efficiencies are possible if the large bandwidths are sacrificed as there usually is a trade-off between effective nonlinearity and conversion bandwidth.

\section{REFERENCES}

[1] M.M. Fejer, G.A. Magel, D.H. Jundt, and R.L. Byer: IEEE J. Quantum Electron., vol. 28, pp. $2631,1992$.

[2] P.G. Kazansky and V. Pruneri: J. Opt. Soc. Am. B, vol. 14, pp. 3170, 1997.

[3] A. Arraf and C.M. de Sterke: IEEE J. Quantum Electron., vol. 34, pp. 660, 1998.

[4] N.E. Yu, J.H. Ro, M. Cha, S. Kurimura, and T. Taira: Opt. Lett., vol. 47, pp. 1046, 2002.

[5] S. Ashihara, T. Shimura, and K. Kuroda: J. Opt. Soc. Am. B, vol. 20, pp. 853, 2003.

[6] D. Faccio A. Busacca, W. Belardi, V. Pruneri, P. Kazansky, T. Monro, D. Richardson, B. Grappe, M. Cooper, and C. Pannell: Electron. Lett., vol. 37, pp. 107, 2001.

[7] A. Ferrando, E. Silvestre, J. Miret, and M. Andrés: Opt.Express, vol. 9, pp. 687, 2000.

[8] J. Lægsgaard and A. Bjarklev: J. Opt. A Pure Appl. Opt., vol. 6, pp. 1, 2004.

[9] T.M. Monro, V. Pruneri, N.G.R. Broderick, D. Faccio, P.G. Kazansky, and D.J. Richardson: IEEE Photon. Tech. Lett., vol. 13, pp. 981, 2001.

[10] S. Johnson and J. Joannopoulos: Opt. Express, vol. 8, pp. 173, 2001.

[11] J.W. Fleming: Electron. Lett., vol. 14, pp. 326, 1978.

[12] J. Lægsgaard, A. Bjarklev, and S. Libori: J. Opt. Soc. Am. B, vol. 20, pp. 443, 2003.

[13] N. Myrén, M. Fokine, O. Tarasenko, L.-E. Nilsson, H. Olsson, and W. Margulis: J. Opt. Soc. Am. B, vol. 21, pp. 2085, 2004.

[14] Y. Kodama and A. Hasegawa: IEEE J. Quant. Electr., vol. QE-23, pp. 510, 1987.

[15] S. Kielich: IEEE J. Quant. Electr., vol. 5, pp. 562, 1969.

[16] B.T. Kuhlmey, R.C. McPhedran, and C.M. de Sterke: Opt. Lett., vol. 27, pp. 1684, 2002.

[17] M.A. van Eijkelenborg, M.C.J. Large, A. Argyros, J. Zagari, S. Manos, N.A. Issa, I. Bassett, S. Fleming, R.C. McPhedran, C.Martijn de Sterke, and N.A.P. Nicorovici: Opt. Express, vol. 9, pp. 319-327, 2001.

[18] J. Jensen, P. Hoiby, G. Emiliyanov, O. Bang, L. Pedersen, and A. Bjarklev: Opt. Express, vol. 13, pp. 5883-5889, 2005. 